\title{
Effectively and accurately mapping global biodiversity patterns for different regions and taxa
}

\author{
Alice Hughes ${ }^{1}$, Michael Orr ${ }^{2}$, Qinmin Yang ${ }^{3}$, and Huije Qiao ${ }^{2}$ \\ ${ }^{1}$ Xishuangbanna Tropical Botanical Garden \\ ${ }^{2}$ Institute of Zoology Chinese Academy of Sciences \\ ${ }^{3}$ Zhejiang University
}

August 4, 2020

\begin{abstract}
Understanding patterns of biodiversity is crucial for developing appropriate conservation and management plans. The IUCN RedList is looked upon as a source of globally-consistent assessment of species extinction risk, including range maps as part of the extinction risk assessment. Species ranges are a central criterion in determining extinction vulnerability, and consequently apportioning conservation and research efforts. Thus, the accuracy of these maps is crucial to the effective conservation of global biodiversity. Given difficulties in acquiring sufficient, reliable point data and the need for species or diversity maps within many studies, countless papers rely on these centralized expert range maps. However, such efforts are vulnerable to errors if not carefully checked, and the drive to assess as many species as possible rather than to ensure meaningful quality assessment may drive high error rates, with huge implications for species conservation. Recent efforts to account for the over-generalization of species ranges by trimming species ranges with landcover and elevation also makes a number of assumptions on the consistency and accuracy of global data, the lack of politically-driven biases. Here, we analyse the biases present in 50768 animal IUCN and BirdLife maps and provide suggestions on how such analyses could be improved, and flag spatial and taxonomic inconsistencies to enable analysis to acknowledge the limitations of data in further analysis based on these maps. We also discuss effective ways to overcome these biases, the limits of such applications and explore alternative means of mapping diversity patterns.
\end{abstract}

\section{Introduction}

Understanding species distributions is fundamental for conservation and management. Accurate maps of species distributions enables assessment of conservation priorities worldwide, based on diversity, threat or endemism, facilitating the development of targeted conservation strategies. Thus, to perform such prioritizations or gap analyses, the spatial accuracy of species ranges is crucial. In the last two decades, IUCN and similar species range maps have become a global currency for the assessment of species distributions across scales and subsequent conservation funding (Jetz et al. 2012, Jenkins et al. 2013, Brooks et al. 2019). Though these maps have been created for varying purposes by different groups, they are now used for global biodiversity analyses, and little has been done to test whether they are truly fit for this purpose.

Species range modeling is highly-sensitive to the data and methods employed. For instance, even basic means of trimming species IUCN ranges using habitat or elevation changes the RedList status of many species via corresponding distributional changes (Ocampo-Peñuela et al. 2016, Li et al. 2017). The reliability of such analyses is crucial not only to apportion adequate and appropriate conservation efforts, but to understand even basic biodiversity patterns, as errors could highlight the wrong areas and species, and with limited resources available this translates to failures to conserve areas and ecosystems critically needing protection.

Unfortunately, accurate and reliable data are rare for most taxonomic groups due to intense sampling biases (Hughes et al. in review). As a consequence, IUCN and similar data underlie countless studies, including 
those on distribution (Buckley \& Jetz 2008, Holt et al. 2013, Rissler \& Smith 2010), climate change (Akcakaya et al. 2006, Sandel et al. 2011, Sekerciuglu et al. 2012), and especially conservation (Jenkins et al. 2013, La Saout et al. 2013, Jetz et al. 2014, Pouzols et al. 2014, Jenkins et al. 2015, Meyer et al. 2015, Runge et al. 2015, Ocampo-Peñuela et al. 2016, Betts et al. 2017, Li et al. 2017, Moran \& Kanemoto 2017, Brooks et al. 2019, Mason et al. 2020). These include analyses at varying resolutions, though $10 \mathrm{~km}^{2}$ is a common resolution since these data are made available for download and analysis (https://biodiversitymapping.org), yet many of these use the maps at a higher resolution than has been shown appropriate Guidance (https://www.conservationtraining.org/enrol/index.php?id=156) states that distribution data should be utilized in developing species range polygons, yet the adherence to these guidelines is not apparent. It is dangerous to use questionable data for real-world conservation, and researchers should carefully assess the limits of the data before applying them to real-world issues.

Here, we test the widespread assumption that the most up-to-date expert range maps (ERMs) (for mammals, odonata, amphibians, birds, and reptiles) provide consistent and standardized species range estimates, exploring whether these range boundaries are associated with administrative divisions. We also assess the proportion of species distribution points for each species falling within their designated IUCN ranges. We discuss and showcase alternative means of mapping diversity patterns, and demonstrate the tradeoff between different approaches, and the limits of applications for each. Understanding biases and inconsistencies helps inform appropriate use and ensures data are fit for purpose, and to make better use of available data to effectively inform research and management decisions based on a clearer understanding of species and richness patterns.

\section{Materials and methods}

Species ranges and boundary density maps

ERMs were downloaded for mammals, odonata and amphibians (https://www.iucnredlist.org/resources/grid/spatialdata); BirdLife maps, and GARD reptile maps (Roll et al. 2017). Each species polygon boundary was converted to a polyline, then split to produce independent polylines per species within each taxa.

To generate species boundary density maps, species were rasterized at $1 \mathrm{~km}$ spatial resolution with the EckertIV projection, and stacked them into a single raster per taxon. The values indicate the number of species whose distribution boundaries overlapped with each cell, enabling overlay with other features to examine overlaps in $\mathrm{R}$. These species boundary density maps underlie most subsequent analyses. $\mathrm{R}$ code, and caveats are given in supplements.

Geographic boundaries

Exploration of species range boundaries suggested numerous geographic datasets were used for different regions and taxa (Ficetola et al. 2014), so analysis was performed for a core set of taxa for all variables, in addition to specialist analysis for groups where other biases were evident.

For all taxa, we assessed the intersection of species range boundaries with national and provincial boundaries, with and without coastal boundaries. An international map was used because international (Western) assessors use them, and do not necessarily denote agreed country boundaries. A $2.5 \mathrm{~km}$ buffer was added on each side of these boundaries in ArcMap to account for potential insignificant deviations from political boundaries.

Species range boundary densities were reclassified into richness classes using the ArcMap quartile function, and the percentage of the top-two, and top-three quartiles of range densities within different buffers $(500 \mathrm{~m}, 1000 \mathrm{~m}, 2500 \mathrm{~m}, 5000 \mathrm{~m})$ calculated per country to determine what percentage of top-quartile data within countries approximated borders. Several buffers were used to assess if there were fewer species range boundaries at greater distances from political boundaries as species ranges may be digitized with differing precisions even if tracking physical or administrative boundaries.

Turnover and administrative boundaries 
Heatmaps were generated by overlaying species ranges per taxon, representing species richness. To assess abrupt diversity changes, standard deviations for $10 \mathrm{~km}$ blocks were calculated using block statistics in ArcMap. Maps were then classified into N=10 categories using the quartile function. Given varied maximum diversity and taxonomic representation, different numbers of richness categories were retained per group, following where borders emerged. This was then extracted using $1 \mathrm{~km}$ buffers of national administrative boundaries to assess percentages of administrative boundaries overlapping turnover hotspots.

Taxon-specific analyses

Data exploration exposed taxon- and regional-specific biases requiring additional analysis. In these cases, the causes of biases were assessed by comparing range boundary density maps to high-resolution imagery and administrative maps via the ArcGis server. These included relationships between 1) amphibians with county borders in the US and 2) dragonflies and river basins globally. In these cases, species boundary density maps were used as a basis to identify potential biases which were then explored empirically using appropriate methods.

For amphibians, counties were digitized using https://gadm.org/ with $2.5 \mathrm{~km}$ buffers. Species boundary density maps for amphibians were reclassified showing where species range boundaries existed with other areas reclassified "no data." Percentages of combined species boundary areas falling within county buffers vs areas without were calculated.

For Odonata, many species were mapped to river basin borders. We used river basins of levels 6-8 in the river hierarchy (https://hydrosheds.org). Two datasets existed for Odonata, the IUCN Odonata specialist group spatial dataset (https://www.iucnredlist.org/resources/spatial-data-download), and a larger dataset available via the RedList website (https://www.iucnredlist.org/resources/grid/spatial-data) containing an additional 1000 polygons relative to the previous file (as of September 2019), predominately in Latin America. We examine both, as either may be used for contemporary analyses on Odonata.

For reptiles, two grids resolutions were visible when mapping species range boundary density (1, 0.5 degrees). Gridding in range delineation was examined by developing 1-, 0.5-degree fishnet grids globally. Grids were then dragged into alignment with the noted reptile range boundary grids in central Africa; if grids are not a genuine artifact of digitization, this would not be possible, or it would be inconsistent in different regions. Alignment between the digitized fishnet grid and range boundaries was reconfirmed in Central Asia and South America. Grids were then clipped to landareas and merged with national boundaries into a combined shapefile. Species range boundary density was quantified and layers reclassified for areas with $>3$ species boundaries overlapping, then intersected with both grid-sizes to quantify percentages of boundary hotspots overlapping with grids or borders.

\section{GBIF cleaning}

GBIF data are useful for testing species distributions. To ensure exclusion of inaccurate localities, we filtered them stepwise before assessing ERMs. Firstly, oceanic records were removed with a terrestrial mask. An ecoregion map (https://ecoregions2017.appspot.com/) was then used to filter samples clearly in the wrong localities using the realms species occupy according to IUCN data. Corrections were made when listed IUCN realms were regularly inconsistent with distribution maps, and further analysis to assay what realm the IUCN range maps were needed assay genuine realms IUCN maps fell into, to develop matching filters and using those realms to filter GBIF data from the same realms.

As GBIF data includes some synonyms, these were also corrected prior to their use. Synonym lists were developed via IUCN-lists, for birds Clements bird checklist (https://www.worldbirdnames.org/ioc-lists/masterlist-2/). As IUCN lists sometimes gave species as both synonyms and true species, any species listed as both was corrected during filtering. Given the slow rate of taxonomic updates on the redlist e.g only $45 \%$ of amphibian species described between 2004- 2016 were assessed by the IUCN (Tapley et al., 2018) and GBIF efforts to update data filters, our approaches are an appropriate resolution and accuracy to assess under-estimation in species ranges from their polygon data. Following GBIF filtering, percentages of points 
within appropriate polygons were calculated. Analyses of relationships between species range boundaries and administrative boundaries (coastal, non-coastal) were made in R.

\section{Exploring alternatives}

Trimming of ERMs by landcover and elevation is regularly promoted as a means to trim ERMs, but it is unknown if simple elevation and landcover trimming correct biases effectively. We tested diversity patterns generated via original ERMs versus those from analysis of bat point data with and without trimming and with published models (Hughes 2017). Point data were clipped for Eurasia and minimum convex polygons (MCPs) created in ArcMap for species with at least five points. Filters were created for each species based on elevation and landcover, both using IUCN assessment data exclusively and that based on extracting environmental data from points, and these were then paired with associated environmental data to clip species range on a per species basis.

We used point data to extract elevation from $1 \mathrm{~km}$-resolution dem, with min, max, mean and standard deviation per species from summary statistics. Species exclusively $<1000 \mathrm{~m}=$ lowland, $1000-2000 \mathrm{~m}=$ mid, $>2000 \mathrm{~m}=$ high, between these ranges ranked accordingly: lowland, low-mid, low-high, mid, high. DEMs were reclassed to corresponding elevation bands. IUCN assessment listings of elevational preference were recorded. A "integrated" status was determined based on comparing the point-based with IUCN-based assessments (when species were assessed in IUCN and had sufficient point data): where only one assessment was given it was retained, where the two agreed it was retained, and where they differed we used the point-based data given higher precision and transparency.

For habitat intactness, we collated IUCN assessments and data extracted from point data. For IUCN assessments we used keywords to assay disturbance tolerance. Habitat listings which referenced roosting in buildings, houses, tunnels were assigned as generalists. Species listed in cultivated areas, paddies, plantations, agriculture were assigned as semi-intact and those listing forest and no other "disturbed" habitats assigned as intact. For point data we classified population layers to under 50 people per kilometer as intact, 51-100 as semi-intact and over 100 per $\mathrm{km}$ as generalist. From point data species with over $50 \%$ of localities in the generalist category were listed as generalists, and species with at least $75 \%$ of records in the under 50 people were classed as intact. The IUCN and point generated categories were then compared, where the two categories differed we selected the "final" classification based on further searches or actual experience with the species listed.

For richness mapping, we joined the elevation field based on species names, split into five elevation categories, each of which was then clipped by a polygon layer of the appropriate elevation bands and merged. This was repeated for the MCP layer and ERM layers. The ERM layer was run twice, for the "integrated" assessment data using the "integrated" category, and once for IUCN elevation assessments. These were then merged to form three species elevation trimmed species collations (one MCP, two ERM). Layers were then joined to intactness categories, and split into three categories prior to trimming with the appropriate intactness filter (intact, semi-intact, generalist). These were then merged before using the count overlap toolbox to count the number of species overlapping in any given area. This enabled comparison of trimmed and untrimmed layers to a previously published Maxent layer (Hughes 2017) to assess how useful these alternate approaches are.

\section{Results}

Overlap between species and administrative boundaries (\%)

Analyses revealed pervasive biases throughout ERMs. For all groups high proportions of species ranges overlapped with administrative borders (Table 1; Figure S1). Non-coastal borders show that administrative boundaries disproportionately impact range limit delineation, averaging $20-30 \%$ of non-coastal species range boundaries coinciding national administrative boundaries alone. Provincial boundaries are also used, increasing the average overlap by upto $10 \%$.

To assess how species turnover relates to administrative areas, we calculated the standard deviations of richness maps (Figure 1), then identified what percentages of national administrative layers were included in 
the higher richness classes, to see what proportion of administrative boundaries fall within these hotspots. For reptiles, the upper four classes were retained, covering $40 \%$ of national boundaries ( $4 \%$ low turnover, $13 \%$ medium turnover $24 \%$ high turnover). This means that the hotspots for reptiles are disproportionately delimited by global administrative boundaries. In total, the overlap between administrative boundaries and these upper classes equates to $37 \%$ of all richness classes, but equals only $10 \%$ of the lowest quartile, $33 \%$ of medium turnover and $82 \%$ of the highest turnover. However, as low-diversity areas will necessarily have low turnover, this was then extracted for areas with $>3$ reptiles species, and for these regions $68 \%$ of national boundaries were on upper richness classes ( $43 \%$ highest turnover).

For amphibians, $34 \%$ of national boundaries overlapped with richness classes (two top richness classes, $27 \%$ high turnover), though equating to only $10 \%$ of high-turnover areas (16\% of the highest levels). For birds, $52 \%$ of national boundaries coincide with turnover (3 upper richness classes, $21 \%$ at highest level), this equates to $29 \%$ of the highest bird turnover. For Mammals, $60 \%$ of national boundaries coincided with high turnover areas (3 top richness classes, $29 \%$ at high turnover), representing $26 \%$ of high turnover areas, and $35 \%$ of highest levels. For Odonata, $40 \%$ of national boundaries were covered (three top richness classes, with $25 \%$ at the highest turnover levels), representing $9 \%$ of high turnover areas but $16 \%$ of the highest.

In terms of individual countries, countries with longer coastlines obviously exhibited higher diversity changes, as coastlines typically mark absolute distributional boundaries. Yet, despite this, some countries with little coastline, and even landlocked countries, showed comparable levels of turnover at political boundaries. For reptiles, Nepal had levels of turnover at political boundaries roughly comparable to that of coastlines, and China also shows exceptional turnover, especially on its southwestern border (Figure 2); Bolivia showed some similar patterns.

Without coastal boundaries included Northern Southeast Asian boundaries are visible across taxa (excepting birds-Figure S1), with the Chinese side of borders show much lower diversity than neighboring Thailand, Vietnam and Myanmar. Iran also shows high turnover along administrative boundaries, considerably below the areas it borders. What is striking is for some countries, especially Southeast Asian countries, $100 \%$ of non-coastal boundaries show overlap with peak areas for species turnover, highlighting the disproportionately large role these borders play in mapping species distribution (Figure S1).

Species turnover on the boundaries

When species ERM boundaries are aggregated to show the number of range boundaries which overlap (Figure $\mathrm{S} 1$ ), and then reclassified to show only areas with the most overlapping species range boundaries, it becomes apparent that the hotspots of where species boundaries are delineated fall on national administrative borders. Within $500 \mathrm{~m}$ of national borders, $30-50 \%$ of the top two richness classes, and 35-50\% of the top three richness classes are included. This increases to between $43-65 \%$ for top-two at $1 \mathrm{~km}$, and $51-70 \%$ of top-three, and $>80 \%$ within $5 \mathrm{~km}$, indicating species range boundaries are frequently delimited at administrative borders, though with varying precision (Table 2).

\section{River basins}

For Odonata, river basins were used by the specialist group to delineate species range limits, and, consequently (Figure S2b), when only the ranges designated by the specialist group are analysed, $>92 \%$ of range boundaries fall on river basin boundaries including a $1 \mathrm{~km}$ buffer (Table S1). This is not the case for newly-added species (largely Latin American), which clearly use a different, less spatially-precise approach, and only $74.3 \%$ of their boundaries fall on river basin boundaries.

\section{US Counties and Amphibians}

Within the USA, county boundaries were primarily used, with only $2 \%$ of the area over $500 \mathrm{~m}$ from county borders containing amphibian range limits, whereas $20 \%$ of county boundaries have $>10$ amphibian species range boundaries recorded, and only $31 \%$ of all US county borders do not show overlap (Figure S2a). Almost $60 \%$ of amphibian species in the US had all their range boundaries on county borders, and the $40 \%$ that show some of their ranges off county borders include invasive alien species like Xenopus laevis . In addition, many 
of these species were range-restricted, with $20 \%$ showing a range $<100 \mathrm{~km}^{2}, 6 \%<10 \mathrm{~km}^{2}$ and $3 \%<1 \mathrm{~km}^{2}$. Notably, similarly low-level administrative areas were not used in other regions.

Reptiles and grids

The gridding of reptile ranges (Figure S2c) is visible in all countries with high reptile diversity (especially tropical and hot areas). Using large (1 degree-111km) grids, the density boundaries included $19 \%$ of boundaries with 3-5 species and $63 \%$ of boundaries with $>5$ species. For all large-grid areas for all global landmasses, $4 \%$ have a minimum of three species boundaries in them, which is surprising as most of the globe is low diversity for species which have been mapped. Smaller $(55 \mathrm{~km})$ grids include $27 \%$ of all boundaries with $3-5$ species, and $71 \%$ of boundaries with five or more species, but is only equivalent to $2.1 \%$ of all small grids across the planet. When this analysis is clipped to areas where three or more species are mapped to occur, these values inflate to $15 \%$ of all large grid area with at least three species, $8.3 \%$ for small $\left(0.5^{\circ}\right)$ grids.

\section{GBIF vs ERMs}

Remarkably many disagreements exist between GBIF and ERM data, with $4 / 5$ taxa having $\sim 1 / 3$ or more of their GBIF point data beyond their corresponding species distribution maps (Table 3a). This obscures differing regional trends, with the Palearctic, Neotropical and Indomalaysian regions containing $>50 \%$ of records outside ERMs on average and the Australasian and Afrotropical regions having between 40-50\% of localities outside their mapped ranges (Table 3b), and others with lower proportions. For species occurring in more than one realm, the number of realms a species is listed in may relate to error, with wider-ranging species showing a higher percentage of localities outside their mapped realms, and reptiles almost uniformly have the lowest accuracy.

\section{Alternative approaches}

In comparing the ERM data to models and polygons derived from the point data, clear differences can be seen (Figure 3). Even untrimmed, MCPs capture the hotspots highlighted by advanced models better than the ERM data, and the ERM maps continue to show clear political borders even when trimmed (Figure 3b, $\mathrm{d}, \mathrm{e}$ ) and too little information exists in the IUCN for the assessment of many species. Following trimming, the MCPs show much higher richness than either of the two trimmed ERM maps, with only a maximum of 18 richness for the ERM data based on the limited species assessments possible using just IUCN data (73 species included- Figure 3d) in bats, upto 70 coexisting species from the "integrated assessment" ERM filtered data (260 species- Figure 3e), and 128 species from the MCPs (340 species- Figure 3f). Simple MCPs not only include many more species, but also recreated the patterns recovered by Maxent and other published studies better than the ERMs (Li et al., 2019).

\section{Discussion}

Expert range maps aim to provide globally consistent and comparable sources of data for mapping biodiversity and endangerment scalably, comparably and representatively. Yet, for all groups examined, the data fail to meet even these basic expectations. Stemming from their differing designs, both within and between groups, there are obvious methodological changes, biases and inconsistencies. Consequently, comparing analyses meaningfully, especially between regions or taxa, may not be possible.

\section{Spatial biases}

Biases in ERM data were inconsistent across space and taxa. Relative to their global area, provincial and country boundaries played disproportionate roles in delineating species ranges, especially in mammals and amphibians. Political boundaries near temperate-tropical transitions in particular have high levels of bias, including borders in South China and northern Southeast Asia (Figure 2, Figure S1), as well as the southwestern Brazil, making the use of these data for these areas exceptionally risky. In these cases, careful assessment for the possibility of strong administrative biases is needed, as using ERMs at these transitions may cause significant errors in analysis, such that alternate approaches such as models or trimmed MCPs should be used where such data exist. 


\section{Comparability across and within groups}

Amphibian, dragonfly and reptile maps have separate drivers of spatial bias, and their biases differ both spatially and temporally as a consequence of differing methodologies. Consequently, the main benefit of using a single data source is entirely lost, as this inconsistency precludes attributing differences between regions or taxa to genuine differences rather than to methodological differences. For example, reptile distribution maps frequently relied on either one-degree or half-degree grids, with these boundaries clearly detectable on maps of species range boundaries and turnover, with, for example, $>71 \%$ of range boundaries shared by $>5$ species falling on either a political boundary or on a half-degree grid. The fact that grids and political areas and other features are variably used only further complicates these issues. Even odonata, where more biologically-relevant river basin boundaries are used, the near-universal use of such features (i.e. 92\% of formerly mapped ranges are on river basin boundaries) may still result in inaccurate maps with both type one and type two errors. Thus, whilst trimming ERMs with appropriate filters could be applied to most taxa, assessments of gridding for reptiles before such an approach could be usefully applied. For other taxa, once possible political boundaries have been assessed, it may be possible to trim species ranges based on clear assessments of habitat needs.

Regional biases are an especially notable issue. For example, only $31 \%$ of US county boundary area (width $=1 \mathrm{~km}$ ) has no amphibian boundary data, whereas $98 \%$ of the land more than $500 \mathrm{~m}$ from a boundary has no amphibian range boundaries (Figure S2a). This is because of a specific initiative within the US, and such inconsistent standards makes comparable analysis between regions impossible (Blackburn et al., 2002). Once US Amphibians which have all their borders on county borders are removed, only $40 \%$ of species remain, and the remainder includes range-restricted and invasive species. As county limits do not typically follow ecological boundaries, they clearly do not represent species boundaries, thus, most forms of trimming could not be used to accurately map ranges or diversity of such a group.

In many cases, the development of published IUCN distributions contradicts their general guidance (IUCN guidelines: https://www.iucnredlist.org/resources/guidelines-for-appropriate-uses-of-red-list-data). Despite the fact that former IUCN training material (https://www.iucn.org/content/iucn-red-list-training-coursenow-online) explicitly said not to draw buffers around single locality points for building distributions, this has clearly been done for hundreds of species across taxa, extensively for some groups (Figure S2d). In addition to other inconsistent biases both within and across groups, these datasets lose the invaluable comparability that would normally be expected from methods that are standardized temporally, spatially and taxonomically. Consequently, further validation and refinement with verified point data is necessary before these data are used for formal conservation management.

\section{Missing the point - errors of omission need solving}

The pervasive biases in these datasets are not a new issue. Range overestimations have been explored (Brooks et al. 2019), but errors of omission have received comparatively little focus. Researchers seemingly assume that, because models overestimate habitat suitability within their borders, the total area of inhabitance should also be excessive. However, a recent analysis ( $\mathrm{Li}$ et al., 2019) found that though BirdLife range maps were typically around ten times the area of predictive models, ERMs still missed areas with recorded, verified records, potentially highlighting areas of limited value whilst missing key areas species may require to survive. We found the same issue in our analyses; even in the best-studied group (birds), $25 \%$ of records fall outside individual ERMs on average, and up to $46 \%$ of records for other groups. This varies significantly between regions, with up to $90 \%$ of records for reptiles in oceanic zones potentially falling outside their ERMs. When we average each region, rather than all data (which emphasizes better-sampled regions in the West), the average accuracy of the results drops for most groups such that many mapped ranges are spatially incorrect and may, thus, misdirect conservation efforts. Altogether, these issues can lead to entirely incorrect estimates of biodiversity hotspots, as seen when comparing the validated models (Hughes, 2017) which showed ERM hotspots were much larger (upto $40 \%$ of the region for some groups relative to upto $5 \%$ for models: Li et al., 2019). Despite this, ERMs still failed to capture $15 \%$ of the most diverse hotspots according to models, and may actively hinder effective conservation efforts by both over-estimating some 
and missing other key biodiversity regions. Thus, at least in developing and tropical regions, these errors in ERMs mean that aggregating distribution data and trimming MCPs based on environmental factors may more accurately map species ranges and have a lower probability of omission than most groups.

Critical hotspots in transboundary areas are particularly likely to be overlooked and missed due to these issues, as some borders show particularly high purported species turnover based on ERM analyses (Figure 2, Figure S1). These issues only further complicate pre-existent challenges with working in close vicinity to many political boundaries. Already, transboundary conservation assessments and recommendations are being made based on these data (Mason et al. 2020). These concerns are even more important for rare and range-limited species, which cannot necessarily be protected merely by conserving more charismatic megafauna. One consequence is that the largest groups are often removed from analyses, for example, some analyses have used only $8 \%$ of mammal species (Visconti et al. 2016), the rough equivalent of assessing plants without angiosperms. Further efforts are needed to increase the breadth and empiricism underlying ERMs and to ensure that data-driven analyses are applied to remove such biases.

\section{Finding a reliable and accurate solution}

Given the difficulty of acquiring verified point data across all species, many researchers modify ERMs to justify mapping diversity at higher resolutions or "accuracy." A common method is to trim species ranges by habitat and elevational range (Li et al., 2016, Ocampo-Peñuela et al. 2016, Brooks et al., 2019). Yet, to do so universally makes a number of critical errors and fails to address the root causes of issues in such analyses.

Firstly, these new approaches do not account for spatial biases associated with administrative boundaries, or missing key range areas (highlighted above). In addition, though refining by habitat is sensible if species are well-known, such data exist for relatively few species. As an example, clipping by altitude makes assumptions about both the level of knowledge on species ranges (defining species' true elevational ranges is challenging for most, with high uncertainty) and the consistency of the data across species ranges (as there is likely to be a relationship between elevation range and latitude for most species with large ranges, and these may shift seasonally); basically, ranges will vary by latitude and may not be known for most species. Additionally, current minimum ranges do not necessarily represent climate-based ecophysiological thresholds for species, as lower-elevation range limits are most vulnerable to being converted to other land-use types and other disturbances, though our point data showed that many species listed as mid or high elevation by the IUCN had also been recorded at sea-level, thus, current IUCN assessments may overlook areas of the range without evaluating what data is available. Our analysis showed that species ranges regularly fell over a much broader area than in ERM assessments even when such a listing was given, and that estimates of range without sufficient data falsely represent true species ranges. Thus, basing future projections on thresholds generated by ERMs will over-inflate species' perceived vulnerability by effectively removing higher-temperature areas that may actually be optimal for some species.

For these reasons, clipping an "expert-generated map" with "expert knowledge on species ranges" may amplify biases, and data-driven alternatives with sensible uncertainty measures should be developed, especially given that most range maps clearly disobey current guidance. A better approach is to sensibly use data of species localities to develop predictions of where species are known to occur, restrict extrapolation to the country or island where localities exist and use these predicted ranges as a replacement for "expert range maps." Such initiatives could also be used to drive data aggregation and sharing, maximizing the availability of open data such as that made available through GBIF (though careful error checking is needed, Orr et al., in review). To further grow these resources, mechanisms must also be developed to better fund taxonomic data verifications and museum data digitization, including also mandated data sharing for projects receiving this funding (Orr et al 2020). Such data are more likely to capture less-accessible areas and rarer species than that generated by citizen scientists, making them invaluable for generating a representative view of the natural world (Hughes et al., in review).

Here, we show that ERMs are biased and inconsistently delineated across space and taxa, and that even simple approaches like minimum convex polygons produce more realistic diversity models. Trimming, which 
can be streamlined into a reproducible approach and applied in a standardized way across species (Figure 3), further improves the performance of MCPs, whilst many shortcomings in ERMs are not ameliorated. As a basis for regional conservation, the data now exist for many taxa to have such data driven approaches, whilst continued use of range maps without careful bias management could misdirect conservation attention.

\section{Looking forwards}

As a scientific community, we should have moved past manually drawing maps of where we assume species are, yet such maps still form the basis of almost all global and regional assessments on biodiversity. Here, we demonstrate that inherent and inconsistent biases within and between these datasets arise from the use of administrative or other convenient boundaries to demarcate species limits, missing up to half the records of many species. Such approaches will lead to incorrect assessments of species vulnerability, potentially highlighting the wrong areas for conservation or management for both single species and communities, especially where transitioning from tropical to subtropical or temperate areas.

Whilst these ERMs were adequate when data were unavailable, initiatives for data digitization and sharing are finally gaining traction as more high-resolution satellite data. Methodological approaches that enable the modelling of species ranges based on data-driven approaches like those shown here are the obvious choice ahead. Whilst expert knowledge has a crucial role in developing and testing such analyses and developing standard frameworks to ensure outputs are meaningful, we have reached a point where we can begin developing truly automated, standardized approaches to inform conservation. We can no longer rely on inconsistent or biased datasets, as doing so limits efforts to digitize real point data and develop new approaches, and conservation advice should no longer rely entirely on these types of expert data alone.

\section{Acknowledgements}

ACH was supported by Chinese National Natural Science Foundation (Grant \#:U1602265, Mapping Karst Biodiversity in Yunnan), the Strategic Priority Research Program of the Chinese Academy of Sciences (Grant No.XDA20050202), the High-End Foreign Experts Program of Yunnan Province (Grant \#:Y9YN021B01, Yunnan Bioacoustic monitoring program), the CAS 135 program (No. 2017XTBG-T03), and the Chinese Academy of Sciences Southeast Asia Biodiversity Research Center fund (Grant \#:Y4ZK111B01). MCO was supported by the Strategic Priority Research Program of the Chinese Academy of Sciences (XDB310304), the NSFC International Young Scholars Program (31850410464) and the CAS President's International Fellowship Initiative (2018PB0003, 2020PB0142). HJQ was supported by the NSFC (31772432).

\section{References}

Akçakaya, H. R., Butchart, S. H., Mace, G. M., Stuart, S. N., \& Hilton-Taylor, C.. (2006). Use and misuse of the IUCN Red List Criteria in projecting climate change impacts on biodiversity. Global Change Biology , 12 (11), 2037-2043.

Alhajeri, B. H., \& Fourcade, Y. (2019). High correlation between species-level environmental data estimates extracted from IUCN expert range maps and from GBIF occurrence data. Journal of Biogeography , 46 (7), 1329-1341.

Betts, M. G., Wolf, C., Ripple, W. J., Phalan, B., Millers, K. A., Duarte, A., .. \& Levi, T. (2017). Global forest loss disproportionately erodes biodiversity in intact landscapes. Nature , 547 (7664), 441.

Blackburn, L., Nanjappa, P. \& Lannoo, M.J. (2002) US Amphibians Distribution Maps. Ball State University and Muncie Center for Medical Education, Indiana University School of Medicine, Muncie, Indiana

Brooks, T. M., Pimm, S. L., Akçakaya, H. R., Buchanan, G. M., Butchart, S. H., Foden, W., .. \& \& Li, B. V. (2019). Measuring terrestrial area of habitat $(\mathrm{AOH})$ and its utility for the IUCN red list. Trends in Ecology 8 Evolution. 34 (11), 977-986.

Buckley, L. B., \& Jetz, W. (2008). Linking global turnover of species and environments. Proceedings of the National Academy of Sciences , 105 (46), 17836-17841. 
Clausnitzer, V., Kalkman, V. J., Ram, M., Collen, B., Baillie, J. E., Bedjanič, M., .. \& Karube, H. (2009). Odonata enter the biodiversity crisis debate: the first global assessment of an insect group. Biological Conservation, 142 (8), 1864-1869.

Ficetola, G. F., Rondinini, C., Bonardi, A., Katariya, V., Padoa-Schioppa, E., \& Angulo, A. (2014). An evaluation of the robustness of global amphibian range maps. Journal of Biogeography , 41 (2), 211-221.

Holt, B. G., Lessard, J. P., Borregaard, M. K., Fritz, S. A., Araújo, M. B., Dimitrov, D., .. \& \& Nogués-Bravo, D. (2013). An update of Wallace's zoogeographic regions of the world. Science, 339 (6115), 74-78.

Hughes, A. C. (2017). Mapping priorities for conservation in Southeast Asia. Biological Conservation, 209 , 395-405.

Hughes, A. C., Orr, M. C., Ma, K., Costello, M. J., Waller, J., Provoost, P., Zhu, C., \& Qiao, H. (In review). Sampling biases shape our view of the natural world. National Science Review

Hurlbert A. H. \& Jetz W. 2007 Species richness, hotspots, and the scale dependence of range maps in ecology and conservation. Proceedings of the National Academy of Sciences 104 (33), 13384-13389

Jenkins, C. N., Pimm, S. L., \& Joppa, L. N. (2013). Global patterns of terrestrial vertebrate diversity and conservation. Proceedings of the National Academy of Sciences , 110 (28), E2602-E2610.

Jenkins, C. N., Van Houtan, K. S., Pimm, S. L., \& Sexton, J. O. (2015). US protected lands mismatch biodiversity priorities. Proceedings of the National Academy of Sciences , 112 (16), 5081-5086.

Jetz, W., McPherson, J. M., \& Guralnick, R. P. (2012). Integrating biodiversity distribution knowledge: toward a global map of life. Trends in Ecology 83 Evolution, 27 (3), 151-159.

Jetz, W., Thomas, G. H., Joy, J. B., Redding, D. W., Hartmann, K., \& Mooers, A. O. (2014). Global distribution and conservation of evolutionary distinctness in birds. Current Biology ,24 (9), 919-930.

Le Saout, S., Hoffmann, M., Shi, Y., Hughes, A., Bernard, C., Brooks, T. M., .. \& R Rodrigues, A. S. (2013). Protected areas and effective biodiversity conservation. Science, 342 (6160), 803-805.

Li, B. V., Hughes, A. C., Jenkins, C. N., Ocampo-Penuela, N., \& Pimm, S. L. (2016). Remotely sensed data informs Red List evaluations and conservation priorities in Southeast Asia. PLOS ONE ,11 (8), e0160566.

Li, J., Hughes, A. C., \& Dudgeon, D. (2019). Mapping wader biodiversity along the East Asian-Australasian flyway. PLOS ONE, 14 (1), e0210552.

Mason, N., Ward, M., Watson, J. E. M., Venter, O., \& Runting, R. K. (2020). Global opportunities and challenges for transboundary conservation. Nature Ecology 83 Evolution . 4 , 694-701

Meyer, C., Kreft, H., Guralnick, R., \& Jetz, W. (2015). Global priorities for an effective information basis of biodiversity distributions. Nature Communications , 6 , 8221 .

Moran, D., \& Kanemoto, K. (2017). Identifying species threat hotspots from global supply chains. Nature Ecology \& Evolution, 1 (1), 0023.

Ocampo-Peñuela, N., Jenkins, C. N., Vijay, V., Li, B. V., \& Pimm, S. L. (2016). Incorporating explicit geospatial data shows more species at risk of extinction than the current Red List. Science Advances ,2 (11), e1601367.

Orr, M. C., Ascher, J. S., Bai, M., Chesters, D., \& Zhu, C. D. (2020). Three questions: How can taxonomists survive and thrive worldwide? Megataxa , 1 (1), 19-27.

Orr, M. C., Hughes, A. C., Chesters, D., Pickering, J., Zhu, C.D., \& Ascher, J. S. (In review). Global patterns and drivers of bee distribution. 
Pouzols, F. M., Toivonen, T., Di Minin, E., Kukkala, A. S., Kullberg, P., Kuusterä, J., .. \& Moilanen, A. (2014). Global protected area expansion is compromised by projected land-use and parochialism.Nature , 516 (7531), 383.

Rissler, L. J., \& Smith, W. H. (2010). Mapping amphibian contact zones and phylogeographical break hotspots across the United States. Molecular Ecology , 19 (24), 5404-5416.

Roll, U., Feldman, A., Novosolov, M., Allison, A., Bauer, A. M., Bernard, R., .. \& \& Colli, G. R. (2017). The global distribution of tetrapods reveals a need for targeted reptile conservation. Nature Ecology \& Evolution , 1 (11), 1677-1682.

Runge, C. A., Watson, J. E., Butchart, S. H., Hanson, J. O., Possingham, H. P., \& Fuller, R. A. (2015). Protected areas and global conservation of migratory birds. Science, 350 (6265), 1255-1258.

Sandel, B., Arge, L., Dalsgaard, B., Davies, R. G., Gaston, K. J., Sutherland, W. J., \& Svenning, J. C. (2011). The influence of Late Quaternary climate-change velocity on species endemism. Science , 334 (6056), 660664.

Şekercioğlu, Ç. H., Primack, R. B., \& Wormworth, J. (2012). The effects of climate change on tropical birds. Biological Conservation, 148 (1), 1-18.

Tapley, B., Michaels, C. J., Gumbs, R., Böhm, M., Luedtke, J., Pearce-Kelly, P., \& Rowley, J. J. (2018). The disparity between species description and conservation assessment: A case study in taxa with high rates of species discovery. Biological conservation, 220, 209-214.

Visconti, P., Bakkenes, M., Baisero, D., Brooks, T., Butchart, S. H., Joppa, L., .. \& Maiorano, L. (2016). Projecting global biodiversity indicators under future development scenarios. Conservation Letters , 9 (1), $5-13$.

Tables

\begin{tabular}{lllllll}
\hline & country & prov & $n c_{-}$count 500 & $n c_{-}$count 1000 & $n c_{-}$count 2500 & nc_count 5000 \\
\hline Amphibia & 26.9 & 31.0 & 9.7 & 12.9 & 16.2 & 21.8 \\
Birds & 35.1 & 43.5 & 15.4 & 20.2 & 23.5 & 26.6 \\
Mammals & 27.9 & 36.8 & 15.0 & 19.9 & 23.3 & 26.5 \\
Odonata & 23.7 & 33.3 & 12.1 & 15.6 & 17.6 & 21.2 \\
Reptiles & 39.2 & 44.0 & 16.1 & 21.1 & 24.8 & 29.4 \\
\hline
\end{tabular}

Table 1. Percentage overlap between species boundaries and administrative boundaries with different buffers sizes (in metres). Country and Province give boundary totals, NC indicates non-coastal boundaries.

\begin{tabular}{lllll}
\hline c2 & $b 500$ & $b 1000$ & $b 2500$ & $b 5000$ \\
\hline Amphibians & 46 & 65 & 74 & 76 \\
Birds & 30 & 44 & 54 & 59 \\
Mammals & 38 & 55 & 64 & 68 \\
Odonata & 48 & 54 & 57 & 61 \\
Reptiles & 30 & 43 & 54 & 58 \\
\hline
\end{tabular}




\begin{tabular}{lllll}
\hline c3 & b500 & b1000 & b2500 & b5000 \\
\hline Amphibians & 40 & 56 & 64 & 68 \\
Birds & 43 & 63 & 74 & 77 \\
Mammals & 48 & 68 & 79 & 82 \\
Odonata & 45 & 52 & 57 & 62 \\
Reptiles & 35 & 51 & 62 & 66 \\
\hline
\end{tabular}

$B$

Table 2a-b. Percentage of all species range boundary hotspots (top-2 and top-3 richness classes of turnover) included within buffers of National administrative boundaries. A greater proportion are captured when only the highest turnover boundaries are used.

\begin{tabular}{lll}
\hline & $P_{-}$in & $P_{-}$out \\
\hline$A$ & 53.56 & 46.44 \\
$B$ & 75.37 & 24.63 \\
$M$ & 66.24 & 33.76 \\
$O$ & 68.52 & 31.48 \\
$R$ & 64.76 & 35.24 \\
\hline
\end{tabular}

Table 3a. Percentage of GBIF points for each species on average for each species inside and outside the IUCN species range boundaries.

\begin{tabular}{llllllll}
\hline Realm & bird_perou & od_perou & rep_perou & mam_perou & amp_perou & all_perou \\
\hline Average & 39 & 59 & 70 & 39 & 52 & 52 \\
Palearctic & 51 & 68 & 62 & 51 & 33 & 53 \\
Neotropical & 39 & 43 & 73 & 39 & 57 & 50 \\
Indomalayan & 27 & 66 & 77 & 27 & 53 & 50 \\
Afrotropical & 23 & 73 & 71 & 23 & 46 & 47 \\
Australasian & 38 & 24 & 65 & 38 & 48 & 43 \\
Nearctic & 52 & & 55 & 52 & 36 & 39 \\
Oceania & 30 & & 90 & 30 & & 30 \\
\hline
\end{tabular}

Table 3b. Percentage of range points outside the IUCN boundaries for each species for each of the realms examined

Figures 




Figure 1. Hotspots of richness based on IUCN data for each taxa. Gridding for reptile data is clearly visible in Africa, and US county boundaries are visible in Amphibian data whilst provinces for India and Australia show in Odonata, Figure S2 provides a higher resolution version of these issues.. 

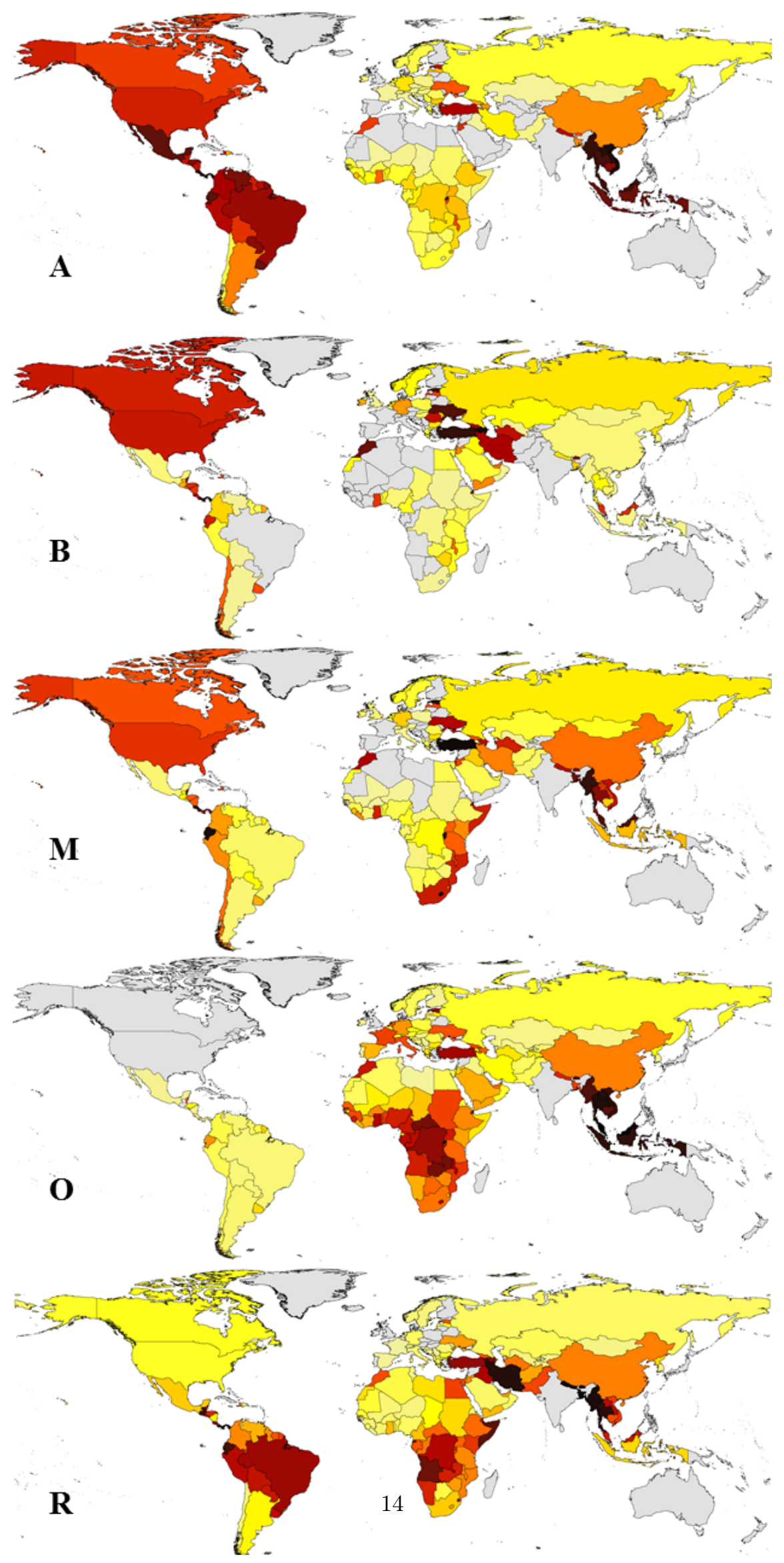
Figure 2. Percentage overlap between richness classes denoting changes in species richness and non-coastal administrative boundaries. $A=$ amphibians, $B=$ birds, $M=$ mammals, $O=$ Odonata, $R=$ reptiles. Grey areas show islands, or areas where high turnover was not detected on any administrative boundary. Darker hues indicate a high percentage of overlap between non-coastal boundaries and high levels of turnover in richness.



Figure 3. Different richness maps for bats in Southeast Asia using a variety of approaches and a standardized legend to enable direct comparison. A. Maxent based model on bats, B. IUCN C. Minimum convex polygons (MCPs), D. Filtered IUCN bats for species with sufficient data in IUCN for trimming, E. IUCN bats with an integrated filter based on a compilation of IUCN assessments and point data. F. Filtered MCPs based on integrated assessments.

\section{Supplements}

\begin{tabular}{lll}
\hline & $p_{-}$basin & $p_{-} b_{-}$buffer \\
\hline All & 68.65 & 85.88 \\
Original & 75.06 & 92.12 \\
New & 56.74 & 74.31 \\
\hline
\end{tabular}

Table $\boldsymbol{S 1}$ Percentage of all species range borders for Odonata which fall on, or within $1 \mathrm{~km}$ of river basin boundaries. This splits data into the original dataset from the Odonata specialist group, the updated dataset for the Americas, and the whole dataset combined. 


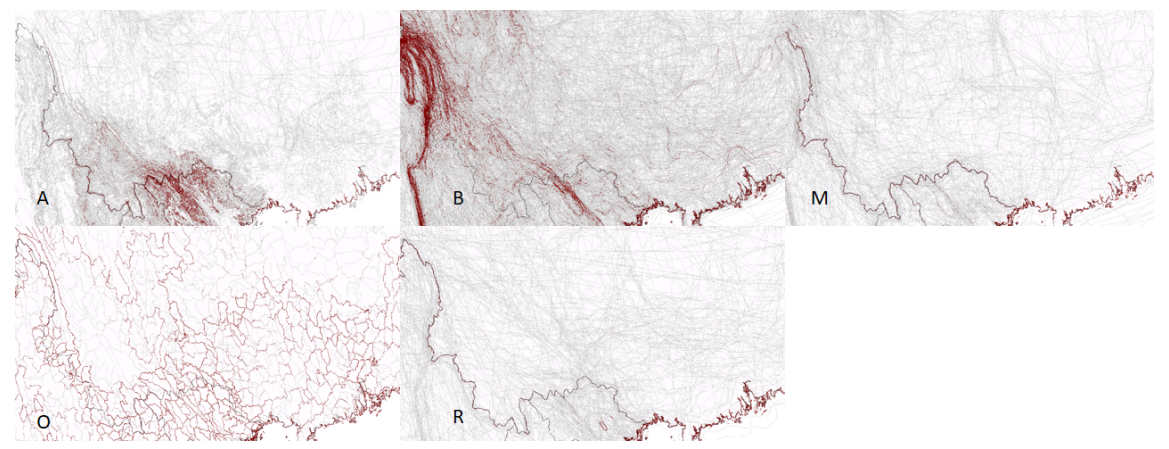

Figure $\mathbf{S 1 .} \quad$ Species range boundary density across all groups around the South China border. $A=$ Amphibians, $B=$ Birds, $M=$ Mammals, $O=$ Odonata, $R=$ Reptiles. Areas with 1-9 borders are shown in grey, those above 10 are shown in dark red. National boundaries are clear for all groups present just based on increased density of species range boundaries at these National boundaries despite the absence of any environmental changes across these regions.

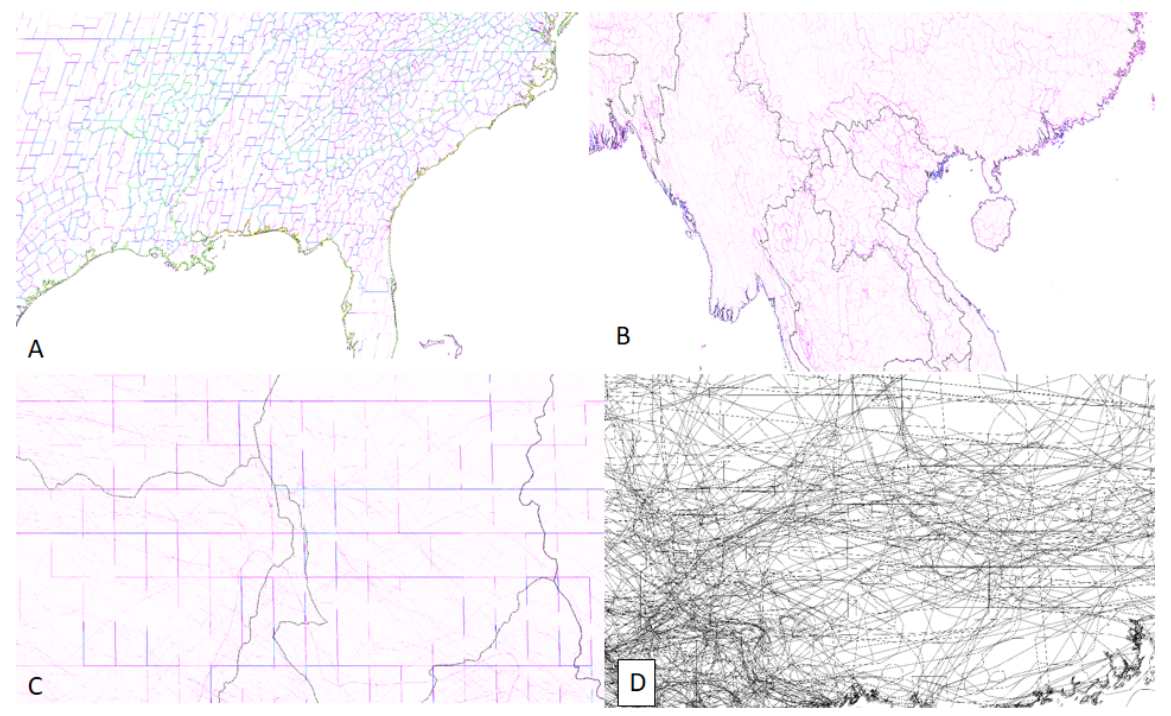

Figure S2. Common errors in different datasets. A-C show density of species range boundaries with increasing hue representing greater numbers of species share range boundaries. A. US Amphibian range boundaries, clearly showing county boundaries. B. Odonata boundaries, both highlighting the river boundaries (viewable globally) and National boundaries. C. Reptile range boundaries showing clear gridding. This image was taken from Africa, but the same patterns are present across the globe. D. Common errors across all datasets showing point buffering at variable distances and no consistent pattern.

Supplemental text

$\mathrm{R}$ code:

GBIF cleaning R v3.6.3 and code is shared via https://github.com/qiaohj/iucn_fix.

Species ranges and boundary density maps $R$ code https://github.com/qiaohj/iucn_fix/blob/master/line2raster_X.r

(replace $\mathrm{X}$ with amphibian, birds, mammals, odonates, reptiles 
Caveats:

In conducting this analysis in must be noted that no single source of data can be expected to be perfect, however researchers must understands the limits of sensible application. GBIF data is acknowledged to include various errors, and whilst they are making continued efforts to clean this data artifacts and other issues are still present. Through the use of filters the impact on such errors should however be minimal.

In terms of buffers, whilst some non-coastal political boundaries do follow mountain ranges, rivers, or other geophysical features; many do not. For example crossing between China and neighbouring countries can often be inadvertent, yet these borders are very clear on many ERM range boundary assessments and their derivatives. Given the lack of physical features for many boundaries it is clear that political and other administrative boundaries are clearly used in delineating ranges. 